\title{
Prospective four years of evaluation of erectile function after low-dose-rate prostate brachytherapy using baseline IIEF-5 > 16
}

\author{
Nadja Schoentgen, MD!, Julien Marolleau, MD',2,3, Francky Delage, MD!, Jean-Baptiste Coquet, MD! \\ Alexandre Fourcade, MD',2,3, Pierre Callerot, MD', Sophie Serey-Eiffel, MD', Jean-Pierre Malhaire, MD4 \\ Olivier Pradier, MD, PhD2,3,4,5, Ulrike Schick, MD, PhD2,3,4,5, Georges Fournier, MD1,2,3,5,6, Antoine Valeri, MD, PhD1,2,3,5,6 \\ 'CHU Brest, Service Urologie, Brest, France, ${ }^{2}$ Université de Brest, Faculté de Médecine et des Sciences de la Santé, Brest, France, ${ }^{3}$ Université \\ Bretagne Loire, Rennes, France, ${ }^{4} \mathrm{CHU}$ Brest, Service de Radiothérapie, Brest, France, ${ }^{5}$ LaTIM, INSERM, UMR IIOI, Univ Brest, Brest, France, \\ ${ }^{b}$ CeRePP, Paris, France
}

\begin{abstract}
Purpose: Prostate brachytherapy (BT) is a validated treatment for localized prostate cancer (CaP) and an attractive therapy option for patients seeking to preserve erectile function (EF). The aim of this paper is to prospectively assess EF evolution during 4 years after BT.

Material and methods: Between February 2007 and July 2012, 179 patients underwent an exclusive Iodine-125 BT, for low-intermediate favorable risk $\mathrm{CaP}$ of whom, $102 \mathrm{had}$ an initial international index of erectile function 5 score $($ IIEF-5) $>16$ and were included in the study. Of those, $12.7 \%$ received neo-adjuvant hormonotherapy (HT) to decrease the prostate volume. Post-BT intake of phosphodiesterase inhibitors (PDE5i) was not an exclusion criterion. Erectile function was prospectively assessed using a validated questionnaire IIEF- 5 before treatment and annually for 4 years.

Results: At 1-year follow-up, 54\% of patients preserved an IIEF-5 $>16$ and only $8 \%$ suffered from severe ED. During the next 3 years, the results were not statistically different. The mean IIEF- 5 lost 4 points during the first year, 17 vs. 21 , and remained stable during the following 3 years. We did not find any significant differences in the proportion of patients treated by PDE5i (18-20\%). As for patients with a normal preoperative IIEF-5 $(>21)(n=52), 35-42 \%$ preserved a normal EF and 71-77\% maintained an IIEF-5 > 16, including 13-19\% of patients who needed PDE5i. Those results were stable for over 4 years.

Conclusions: During the first 4 years after BT, more than half of patients maintained an IIEF-5 > 16, and EF results remained stable. Severe erectile dysfunction (ED) was very rare.

J Contemp Brachytherapy 2019; 11, 3: 195-200 DOI: https://doi.org/10.5114/jcb.2019.85793
\end{abstract}

Key words: prostate cancer, erectile dysfunction, brachytherapy, radiation therapy, IIEF-5.

\section{Purpose}

Prostate cancer $(\mathrm{CaP})$ is the second most common cancer and the fifth leading cause of death from cancer in men in the world [1]. Nowadays, many treatment options available are responsible for side effects, mainly urinary and sexual. During the announcement consultation when the patient is learning about his disease, sexual consequences are often undervalued by the patient who wants to eradicate his cancer. However, once the treatment is implemented, side effects are prominent and impair the quality of life. Life expectancy after curative treatment is more than ten years; therefore, the management of side effects is of paramount importance. Prostate brachytherapy $(\mathrm{BT})$, with oncologically proven effectiveness [2,3], is one of the recommended treatment for patients suffering from low-risk CaP [4]. Erectile dysfunction (ED) after BT with or without external beam radiotherapy (EBRT) has been reported to vary from $19 \%$ to $80 \%$ [5]. Indeed, published studies on ED after BT are very heterogeneous: 1. Different inclusion criteria in populations: high-grade $\mathrm{CaP}$, high-rate of preoperative hormonotherapy $(\mathrm{HT})$, or preoperative ED; 2 . Various methods with different BT techniques; and 3. Difference in the definition and assessment of ED, i.e., patient interview, physician-reported scores, many different questionnaires such as International Index of Erectile Function five item score (IIEF-5), IIEF-15, or Mount Sinai questionnaire [6,7,8,9,10]. In a recent review of Wortel et al. regarding the challenges in interpretation studies on ED after radiation therapy for 
$\mathrm{CaP}$, only 4 studies reported on ED after exclusive lowdose-rate BT were identified, with $24-58 \%$ of patients completing the study at 3-5 years and only 3 patients using an IIEF questionnaire [5].

A recent interesting study has addressed the issue of the impact of radiation therapy (i.e., BT, EBRT, or both) on erectile function (EF) [11]. In their articles review, the authors included 103 studies assessing ED after: BT $(n=17,057$ patients), EBRT $(n=8,166)$, or both $(n=1,046)$, and performed a meta-analysis and meta-regression. The meta-regression showed no difference for ED after BT or EBRT, reaching about $33 \%$ at 1 year and $50 \%$ at 5 years. However, 7 common instruments were used to assess EF with 23 different cutoffs to define ED. The metaanalysis was conducted only on 21 studies that used the IIEF-5, also known as SHIM-questionnaire (sexual health inventory for men), it did not stratify according to RT modality (i.e., EBRT vs. BT), and the pooled analysis used the SHIM/IIEF-5 ED cutoff varying from 10 to 17.

In this context, we aimed to report the prospective evaluation of EF over 4 years after BT in patients with low-risk $\mathrm{CaP}$, using the validated self-administrated questionnaire IIEF-5 in a well-defined EF population at baseline (IIEF-5 > 16) and using the same BT technique.

Table 1. Population characteristics

\begin{tabular}{|c|c|}
\hline Characteristics & Values \\
\hline Mean age (years) & $64.4( \pm 6)$ \\
\hline Mean PSA (ng/ml) & $5.8( \pm 1.9)$ \\
\hline PSA $<10$ & $n=99$ \\
\hline PSA 10-15 & $n=3$ \\
\hline Mean prostatic volume $\left(\mathrm{cm}^{3}\right)$ & $41.7( \pm 11.3)$ \\
\hline \multicolumn{2}{|l|}{ Gleason score } \\
\hline$<7$ & $n=100$ \\
\hline $7(3+4)$ & $n=2$ \\
\hline IIEF-5, mean $( \pm \sigma)$ & $21.4( \pm 2.7)$ \\
\hline IIEF-5 > 21 & $n=52(51 \%)$ \\
\hline IIEF-5 17-21 & $n=50(49 \%)$ \\
\hline \multicolumn{2}{|l|}{ Clinical stage } \\
\hline cT1c & $n=98(96.1 \%)$ \\
\hline cT2a & $n=3(2.9 \%)$ \\
\hline cT2b & $n=1(1 \%)$ \\
\hline Preoperative HT & $n=13(12.7 \%)$ \\
\hline \multicolumn{2}{|l|}{ Comorbidity } \\
\hline Cardiovascular disease & $n=32(31.4 \%)$ \\
\hline Diabetes & $n=11(10.8 \%)$ \\
\hline
\end{tabular}

PSA-prostate specific antigen, IIEF-5-international index of erectile function 5 , HT-hormonotherapy

\section{Material and methods}

This study was a mono-centric retrospective cohort with prospective assessment. From February 2007 to July 2012, 179 patients with localized CaP were treated with BT. Inclusion criteria were the BT eligibility criteria [4]: 1. Low-risk $\mathrm{CaP}$ or intermediate favorable $\mathrm{CaP}$ according to D'Amico classification, i.e., Gleason score $<7$ with $\mathrm{PSA}<10 \mathrm{ng} / \mathrm{ml}$ and $<\mathrm{T} 2 \mathrm{~b}$, or only one of the following criteria: PSA $10-15 \mathrm{ng} / \mathrm{ml}$ or T2b or Gleason $7(3+4)$; 2 . Less than half of positive biopsies; 3 . Initial prostate volume < $50 \mathrm{cc}$ or $80 \mathrm{cc}$ decreased to $50 \mathrm{cc}$ after 3 months preoperative HT; 4 . IIEF-5 $>16$ preoperatively without drug assistance. The exclusion criteria were: 1 . Preoperative IIEF-5 $\leq 16$; 2 . History of pelvic EBRT; 3 . Intaking of phosphodiesterase inhibitor 5 (PDE5i) before treatment of CaP. Patients were not randomized; they have selected their treatment after clear, fair, and appropriate information.

Of the 179 patients treated by BT, 163 completed the initial IIEF-5 questionnaire and among these patients, 103 had IIEF-5 > 16; 1 patient was excluded because of a preoperative PDE5i treatment. Therefore, 102 patients with localized $\mathrm{CaP}$ and having an initial IIEF-5 score $>16$ were included. All patients were sexually active at baseline. Population characteristics are summarized in Table 1. No patient received EBRT and $12.7 \%(n=13)$ had 3 months preoperative HT because of prostate volume $>50 \mathrm{~cm}^{3}$. No cancer recurrence occurred during follow-up.

During the study, PDE5i was introduced after BT at the request of the patient or proposed by the medical team in case of IIEF- $5<16$ at a follow-up visit (sildenafil $100 \mathrm{mg}$, tadalafil on demand $20 \mathrm{mg}$ or $5 \mathrm{mg}$ daily). No patient was treated with intra-cavernous injection, penile implant, or vacuum. IIEF-5 for patients using PDE5i corresponded to the score under treatment. Preoperative HT and PDE5i after BT were not considered as exclusion criteria.

All BT were performed by the same experienced team included a urologist, a radiotherapist, and a physicist. The technique of Iodine-125 $\left({ }^{125} \mathrm{I}\right)$ loose seeds, as described by Stone and Stock, was already published in our previous studies, and was carried out with a prescribed dose of 160 Gy [12,13]. The BT modalities are presented in Table 2.

Erectile function was prospectively assessed by a validated self-administered questionnaire IIEF-5. This questionnaire is the simplified version of 5 items of the IIEF questionnaire, which was developed by Rosen in 1996 [14]. It is recommended as a criterion of effectiveness in clinical studies on ED. It enables to classify patients into 5 classes such as: normal EF: > 21; mild ED: 17-21; mild to moderate ED: $12-16$; moderate ED: 8-11; severe ED: $<8$. A score below 5 is unevaluable. This questionnaire was completed by all patients included in our research before BT (or before HT) and annually for 4 years. An informed consent was obtained from every patient participating in the study. The primary endpoint was the IIEF-5 score. We used the Mann-Whitney test and the Chi-square test to analyze statistical difference between the years of follow-up. 


\section{Results}

A population of 102 patients was analyzed. No CaP recurrence and death from $\mathrm{CaP}$ had occurred, and no patient received adjuvant oncological therapy during follow-up. One patient died at 18 months of follow-up from other cause. At 1 year after BT (Figure 1, Table 3), 98 subjects were analyzed. Among them, 8 were unevaluable (with an IIEF-5 score < 5) due to not having sex for other reasons than ED: 2 because of wife refusal, 1 because of painful ejaculation, 1 because of recent heart failure, and 4 because of no sex partner. Moreover, 18 were treated by PDE5i on demand. Of the 90 evaluable patients, 49 (54\%) preserved an IIEF-5 $>16$, enabling patient to have sexual relationship, and $73(81 \%)$ had IIEF-5 above the score of 12 . Moreover, only $7(8 \%)$ presented a severe ED (score $<8$ ). The average IIEF- 5 score was 17 and lost 4 points during the first year.

During the $2^{\text {nd }}, 3^{\text {rd }}$, and $4^{\text {th }}$ year, the proportion of patients maintaining an IIEF-5 score $>16$ was not statistically different from that observed at the first year $(p=0.94$; range, 60-64\%). There was no statistical difference between the IIEF-5 means during follow-up (17-18). We did not observe any significant difference in the proportion of patients treated by PDE5i $(18-21 \%$; $p=0.85)$. At 2 years, 11 patients were lost to follow-up, one patient died (from other reason than $\mathrm{CaP}$ ), and 10 were not evaluated because of 2 divorces and 8 without sex partner. At 3 years, 12 were lost and 14 were unevaluable because of 4 divorces, 2 wife refusal, and 7 no sexually active, who refused to talk about their sexual life or pretended to no longer be interested in the sexual aspect of life. At 4 years, 24 were lost and 12 were unevaluable with the same previous reasons.

At least a minimum of $54 \%$ and $80 \%$ of patients maintained a score $>16$ and $\geq 12$, respectively. Less than $10 \%$ suffered from severe ED during the 4 years post-BT, and the proportions of subjects belonging to different IIEF- 5 groups remained stable over the 4 years following BT.

A subgroup analysis was performed on patients with preoperative IIEF-5 > 21 (Figure 2, Table 4): 6 patients were lost to follow-up at 4 years and 2 were unevaluable. At 1 year, 52 patients were analyzed (100\%) and 4 had an unevaluable score ( 2 because of wife refusal and 2 no sex partner). Of the 48 evaluable patients with a preoperative IIEF-5 score $>21,35 \%$ preserved a score above 21 , and $75 \%$ preserved a score $>16$. Nine patients were treated by PDE5i and the mean IIEF- 5 score was 18 . Those results were stable over 4 years, with a minimum rate of $35 \%$ keeping a normal $\mathrm{EF}$ and a minimum rate of $70 \%$ keeping a normal EF or mild ED (IIEF-5 > 16).

\section{Discussion}

In this study with a longitudinal evaluation, we included a homogenous population (102 patients) treated with the same BT technique (one team, no external radiation, loose ${ }^{125}$ I seeds). We used a validated self-administrated questionnaire for ED. All patients had IIEF-5 > 16 at baseline and none had preoperative PDE5i treatment. We found that the proportions of subjects classi-
Table 2. Modalities for prostate brachytherapy

\begin{tabular}{|c|c|}
\hline Characteristics & Values \\
\hline Mean number of needle $( \pm \sigma)$ & $22( \pm 2.6)$ \\
\hline Mean number of source $( \pm \sigma)$ & $66.4( \pm 9.9)$ \\
\hline \multicolumn{2}{|l|}{ Mean dose received by: } \\
\hline \multicolumn{2}{|l|}{ Prostate } \\
\hline $\mathrm{D}_{90}( \pm \sigma)$ & 184.7 Gy $( \pm 6)$ \\
\hline $\mathrm{V}_{100}( \pm \sigma)$ & $97.8 \%( \pm 1.9)$ \\
\hline \multicolumn{2}{|l|}{ Rectum } \\
\hline $\mathrm{D}_{2 \mathrm{cc}}( \pm \sigma)$ & 107 Gy $( \pm 15.1)$ \\
\hline $\mathrm{D}_{0.1 c c}( \pm \sigma)$ & $154.1 \mathrm{~Gy}( \pm 18.4)$ \\
\hline$R_{100}( \pm \sigma)$ & 0.12 Gy $( \pm 0.24)$ \\
\hline \multicolumn{2}{|l|}{ Dosimetry constraints } \\
\hline \multicolumn{2}{|l|}{ Urethra } \\
\hline $\mathrm{D}_{10}$ & 218 Gy \\
\hline $\mathrm{D}_{30}$ & $188 \mathrm{~Gy}$ \\
\hline \multicolumn{2}{|l|}{ Rectum } \\
\hline$D_{2 c c}$ & 145 Gy \\
\hline $\mathrm{D}_{0.1 \mathrm{ccc}}$ & $200 \mathrm{~Gy}$ \\
\hline
\end{tabular}

$D_{90}$ - dose received by $90 \%$ of prostatic volume, $V_{100}$ - prostatic volume receiv ing $100 \%$ of the prescribed dose of $160 \mathrm{~Gy}, D_{2 c c}$ - maximal dose received by 2 cc of the rectal volume, $D_{0.1 c c}$ - maximal dose received by $0.1 \mathrm{cc}$ of the rectal volume, $R_{100}$ - dose received by $100 \%$ of the rectal volume, $D_{10}$ and $D_{30}$-dose received by $10 \%$ and $30 \%$, respectively, of the urethral volume

fied in the different IIEF-5 groups remained stable over 4 years following BT, and $63 \%$ maintained an IIEF-5 $>16$ at 4 years. Less than $10 \%$ of patients were suffering from severe ED. Moreover, in the preoperative normal EF group (IIEF-5 $>21$ ), about $70 \%$ of patients preserved a score $>16$ and $35 \%$ had a normal EF.

There are few studies available on EF after exclusive $\mathrm{BT}$, and most of them are retrospective. Additionally, they are very heterogeneous, which makes it difficult to compare. Indeed, the populations are not comparable;

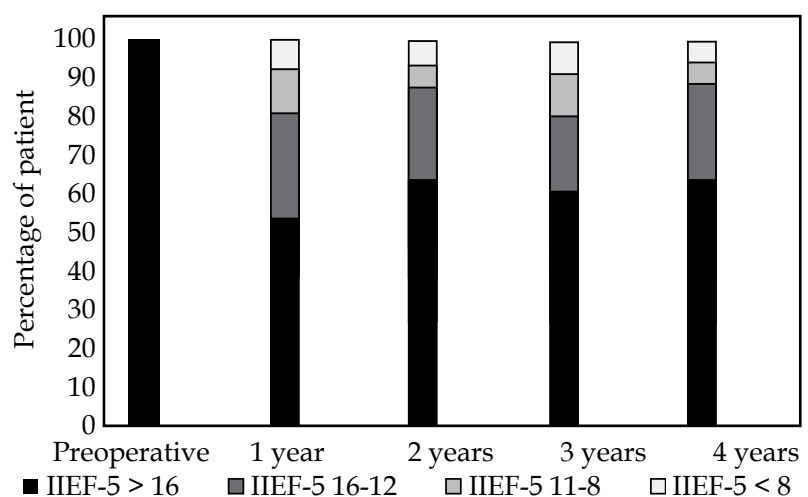

Fig. 1. Evolution of erectile function by IIEF-5 groups over 4 years after brachytherapy 
Table 3. Evolution of erectile function after brachytherapy over 4 years

\begin{tabular}{lcccccccc} 
& $N$ studied (\%) & N evaluable (\%) & IIEF-5 $>16$ & IIEF-5 $16-12$ & IIEF-5 $11-8$ & IIEF-5 $<8$ & Mean IIEF-5 & $n$ PDE5i \\
\hline Preoperative & $102(100 \%)$ & $102(100 \%)$ & 102 & 0 & 0 & 0 & 21 & 0 \\
\hline 1 year & $98(96 \%)$ & $90(88 \%)$ & $49(54 \%)$ & $24(27 \%)$ & $10(11 \%)$ & $7(8 \%)$ & 17 & $18(20 \%)$ \\
\hline 2 years & $90(88 \%)$ & $80(79 \%)$ & $51(64 \%)$ & $19(24 \%)$ & $4(5 \%)$ & $6(7 \%)$ & 18 & $17(21 \%)$ \\
\hline 3 years & $89(87 \%)$ & $75(74 \%)$ & $45(60 \%)$ & $15(20 \%)$ & $8(11 \%)$ & $7(9 \%)$ & 17 & $15(20 \%)$ \\
\hline 4 years & $78(76 \%)$ & $65(64 \%)$ & $41(63 \%)$ & $16(25 \%)$ & $4(6 \%)$ & $4(6 \%)$ & 18 & $12(18 \%)$
\end{tabular}

IIEF-5 - international index of erectile function 5, PDE5i-phosphodiesterase 5 inhibitor

some studies included patients with high-grade $\mathrm{CaP}$ non eligible for BT treatment $[7,10,15]$, and others included patients with preoperative ED $[16,17]$ or high percentage of preoperative HT [7]. The methods of treatment were also heterogeneous, with different BT techniques $[15,18]$, dissimilar questionnaires validated or not [17], and adjuvant treatment sometimes not mentioned. In 2011, Bannowsky et al. [19] published a study on EF after low-dose-rate BT for localized $\mathrm{CaP}$ and found a significant loss of EF in the third year highlighting a delayed effect on EF. However, this study included only 32 patients and no ED treatment after BT was done. Chen et al. described stable sexual scores over 2 years post$\mathrm{BT}$, but they only used the sexual domain of the prostate cancer symptom index score and analyzed only 78 BT patients at 2 years [20]. The Mont Sinai team showed sta-

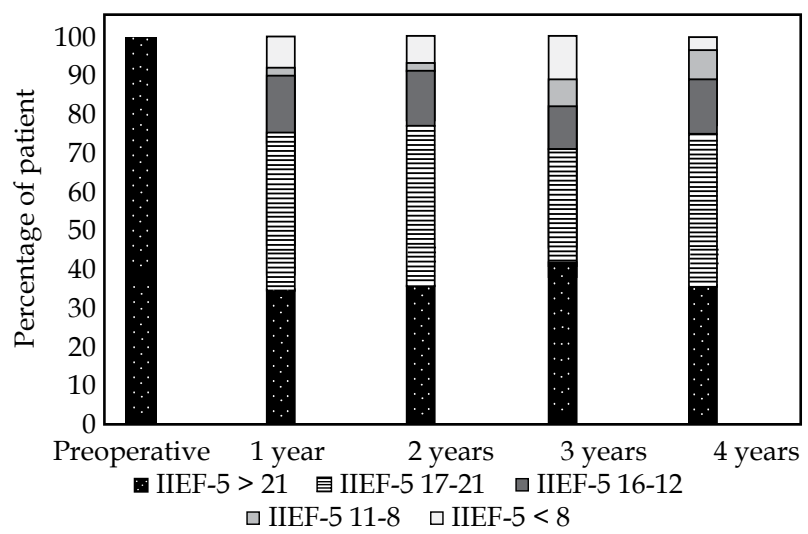

Fig. 2. Evolution of erectile function over 4 years after brachytherapy for patients with preoperative normal IIEF-5 (i.e. $>21$ ) ble results of EF over 5 and 10 years in a large cohort of patients (including different BT technique and various assessment of EF) [15].

We used the IIEF-5, which is a validated, simple, and reproducible questionnaire, recommended as the best tool to assess ED after radiation therapy for $\mathrm{CaP}$ [5]. This questionnaire has been already employed in other studies, but with several inclusion bias such as preoperative ED patients not excluded [16], low number of patients [19], or IIEF-5 not used for all patients [10]. For our study, we selected patients with an IIEF- 5 score $>16$ to avoid a bias due to preoperative ED. A cutoff score of 16 for inclusion of patients and their analysis allowed to obtain a representative sample of the general population and an analysis applicable to everyday practice. In 1998, Rhoden et al. [21] used this score to assess the prevalence of ED in 965 Brazilian patients aged $40-90$ participating in a CaP screening program. In this study, in the 40-49 years old age group (most unaffected by ED), only $64 \%$ of men had a strictly normal EF (IIEF-5 > 21) and $91 \%$ had a score $>16$. In the 60-69 years old age group, only $42 \%$ had a strictly normal EF, while $65 \%$ had a score $>16$. We chose a score above 16 for inclusion, because the mean age of our population was 65 years and, at this age, only $42 \%$ of men have normal EF and $65 \%$ have normal or mild ED [21]. Considering assessment of the results, we also voluntarily chose not to exclude patients under PDE5 inhibitors after BT mainly because it can be considered as a mini-invasive treatment and it's a real-life practice. Moreover, our study fulfilled all the requirements recommended by experts for reporting on erectile function outcomes after radiation therapy for $\mathrm{CaP}$, i.e., methods of patients selection, patients and treatment characteristics, the use and duration of HT, baseline sexual function, the use of validated questionnaire, follow-up $\geq 24$-36 months,

Table 4. Evolution of erectile function over 4 years after brachytherapy for patients with preoperative IIEF-5 $>21$ $N$ studied (\%) $\quad N$ evaluable (\%) IIEF-5 > 21 IIEF-5 17-21 IIEF-5 16-12 IIEF-5 11-8 IIEF-5 < 8 Mean IIEF-5 $n$ PDE5i

\begin{tabular}{|c|c|c|c|c|c|c|c|c|c|}
\hline Preoperative & 52 (100\%) & 52 (100\%) & 52 (100\%) & 0 & 0 & 0 & 0 & 24 & 0 \\
\hline 1 year & 52 (100\%) & $48(92 \%)$ & 17 (35\%) & $19(40 \%)$ & $7(15 \%)$ & $1(2 \%)$ & $4(8 \%)$ & 18 & $9(19 \%)$ \\
\hline 2 years & 48 (92\%) & $44(85 \%)$ & $16(36 \%)$ & $18(41 \%)$ & $6(14 \%)$ & $1(2 \%)$ & $3(7 \%)$ & 18 & $8(17 \%)$ \\
\hline 3 years & $50(96 \%)$ & $45(87 \%)$ & $19(42 \%)$ & $13(29 \%)$ & $5(11 \%)$ & $3(7 \%)$ & $5(11 \%)$ & 17 & $6(13 \%)$ \\
\hline 4 years & $46(88 \%)$ & $44(85 \%)$ & $16(36 \%)$ & $17(39 \%)$ & $6(14 \%)$ & $3(7 \%)$ & $2(4 \%)$ & 19 & $7(16 \%)$ \\
\hline
\end{tabular}

IIEF-5 - international index of erectile function 5, PDE5i - phosphodiesterase 5 inhibitor 
definition and proportion of erectile dysfunction, and the use of erectile aids [5].

A proportion of $54 \%, 64 \%, 60 \%$, and $63 \%$ of patients preserved an IIEF-5 $>16$ at 1, 2, 3, and 4 years, respectively, with no statistical difference between years $(p=0.94)$. Those results were stable over years as observed by Putora et al. (3 years stability of EF after BT) [16]. In the same way, Ong et al. published in 2014 an interesting study on long-term evolution of EF after prostate BT, using the IIEF-5 questionnaire [22]. They reported $59 \%$ of EF preservation at 5 years (median follow-up of 41 months), defined by an IIEF- 5 score $>16$. EF was stable from the first year post-BT. Our study found similar results, but only $18 \%$ of our patients needed PDE5i (40\% in the study by Ong et al.), and we added the subgroup analysis of preoperative IIEF-5 > 21 patients. Moreover, patients treated with PDE5i before BT were not excluded in the Ong et al. study ( $8 \%$ had PDE5i before BT), and only $44 \%$ and $33 \%$ of the entire cohort was assessed at 4 and 5 years, respectively [20].

In our study, a minimum of $80 \%$ of the population presented IIEF-5 score $\geq 12$. Delaunay et al. [23] published a study with 270 patients at 3 years after BT on EF and quality of orgasm after the treatment [23]. They observed that patients with an IIEF- $5 \geq 12$ had quite frequently intense orgasm than those with a score $<12(26.7 \%$ vs. $2.7 \%$; $p<0.001)$. Langley et al. found a proportion of $72 \%$ of patients who preserved potency (IIEF- $5>11$ ) at 5 years [24]. We also noticed that patients with a score of $<8$ represented less than $10 \%$ of patients and among these patients, half of them refused PDE5i treatment and refused to talk about their sexual life or pretended to no longer be interested in the sexual aspect of life.

Some studies on EF after BT also sought to identify other ED risk factors in their population. Merrick et al. identified preoperative IIEF score as predictive factor of ED in a multivariate analysis, and Matsushima et al identified age $\geq 70$ years as ED risk factor $[9,18]$. We performed, at one year follow-up of our study, a multivariate analysis on ED risk factors, which was already the subject of another publication and was not the focus of this study [25].

We then conducted a multivariate analysis on predictive factors of ED with IIEF- $5 \leq 16$ by logistic regression. The analyzed risk factors included: preoperative IIEF-5 score, age, cardiovascular disease, diabetes, preoperative $\mathrm{HT}$, intake of PDE5i, and preoperative prostate volume. Preoperative IIEF-5 score $\leq 21$ was the only independent predictive factor of ED identified in our cohort, with an odd ratio of 6.5 [IC 95\%: 2.4-17.4 $(p=0.0002)]$. None of the other analyzed parameter was identified as ED independent risk factor.

A limitation of our study may be, as observed in most studies on this topic, the proportion of patients lost to follow-up, i.e., $4 \%$ at 1 year, about $11 \%$ at $2-3$ years, and $23.5 \%$ at 4 years. Additionally, in the study of Ong et al. using a large population with IIEF- $5>16$ at baseline, only $58 \%, 44 \%$, and $33 \%$ of patients were assessed at 3, 4, and 5 years, respectively [22]. Furthermore, the lost to follow-up we observed in our study was lower in the subgroup of patients with normal baseline IIEF-5, i.e.,
$0-11.5 \%$. The second limitation is the relatively small size of study group, however, homogenous at different points of view, as mentioned above. We also used a questionnaire that focuses only on EF and does not explore other aspects of human sexuality. A prospective multicenter study using a multimodal questionnaire on sexual life would be interesting to perform in order to have a wider assessment of sexuality in this population.

\section{Conclusions}

Studies on side effects of $\mathrm{CaP}$ treatments are crucial to guarantee a loyal and clear preoperative information and a choice of better treatment option according to patient's request. In our cohort, EF in patients with low-risk cancer treated by BT remained stable during the first four post-operative years, and severe ED affected less than $10 \%$ of patients. This suggests that BT could be one of the primary options to be considered for patients with priority to preserve their EF. This hypothesis should be studied with a prospective multicentric design with assessing the impact of BT over other aspects of sexual life.

\section{Disclosure}

Authors report no conflict of interest.

\section{References}

1. Ferlay J, Soerjomataram I, Dikshit R et al. Cancer incidence and mortality worldwide: Sources, methods and major patterns in GLOBOCAN 2012: Globocan 2012. Int J Cancer 2015; 136: E359-E386.

2. Sylvester JE, Blasko JC, Grimm PD et al. Ten-year biochemical relapse-free survival after external beam radiation and brachytherapy for localized prostate cancer: the Seattle experience. Int J Radiat Oncol Biol Phys 2003; 57: 944-952.

3. Thompson I, Thrasher JB, Aus G et al. Guideline for the management of clinically localized prostate cancer: 2007 update. J Urol 2007; 177: 2106-2131.

4. Mottet N, Bellmunt J, Bolla M et al. EAU-ESTRO-SIOG Guidelines on Prostate Cancer. Part 1: Screening, Diagnosis, and Local Treatment with Curative Intent. Eur Urol 2017; 71: 618-629.

5. Wortel RC, Incrocci L, Mulhall JP. Reporting erectile function outcomes after radiation therapy for prostate cancer: challenges in data interpretation. J Sex Med 2017; 14: 1260-1269.

6. Stock RG, Kao J, Stone NN. Penile erectile function after permanent radioactive seed implantation for treatment of prostate cancer. J Urol 2001; 165: 436-439.

7. Nishimura S, Yorozu A, Ohashi T et al. Five-year potency preservation after iodine-125 prostate brachytherapy. Int J Clin Oncol 2014; 19: 940-945.

8. Taira AV, Merrick GS, Galbreath RW et al. Erectile function durability following permanent prostate brachytherapy. Int J Radiat Oncol Biol Phys 2009; 75: 639-648.

9. Merrick GS, Butler WM, Wallner KE et al. Erectile function after prostate brachytherapy. Int J Radiat Oncol Biol Phys 2005; 62: 437-447.

10. Cesaretti JA, Kao J, Stone NN et al. Effect of low dose-rate prostate brachytherapy on the sexual health of men with optimal sexual function before treatment: analysis at $>$ or $=7$ years of follow-up. BJU Int 2007; 100: 362-367.

11. Gaither TW, Awad MA, Osterberg EC et al. The natural history of erectile dysfunction after prostatic radiotherapy: 
a systematic review and meta-analysis. J Sex Med 2017; 14: 1071-1078.

12. Stone NN, Stock RG. Brachytherapy for prostate cancer: real-time three-dimensional interactive seed implantation. Tech Urol 1995; 1: 72-80.

13. Le Fur E, Malhaire J-P, Nowak E et al. Impact of experience and technical changes on acute urinary and rectal morbidity in low-dose prostate brachytherapy using loose seeds real-time implantation. Brachytherapy 2013; 12: 589-595.

14. Rosen RC, Cappelleri JC, Smith MD et al. Development and evaluation of an abridged, 5-item version of the International Index of Erectile Function (IIEF-5) as a diagnostic tool for erectile dysfunction. Int J Impot Res 1999; 11: 319-326.

15. Snyder KM, Stock RG, Buckstein M et al. Long-term potency preservation following brachytherapy for prostate cancer. BJU Int 2012; 110: 221-225.

16. Putora PM, Engeler D, Haile SR et al. Erectile function following brachytherapy, external beam radiotherapy, or radical prostatectomy in prostate cancer patients. Strahlenther Onkol Organ Dtsch Rontgengesellschaft Al 2016; 192: 182-189.

17. Marina O, Warner J, Ye $\mathrm{H}$ et al. An age-corrected matchedpair study of erectile function in patients treated with dose-escalated adaptive image-guided intensity-modulated radiation therapy vs. high-dose-rate brachytherapy for prostate cancer. Brachytherapy 2014; 13: 163-168.

18. Matsushima M, Kikuchi E, Maeda T et al. A prospective longitudinal survey of erectile dysfunction in patients with localized prostate cancer treated with permanent prostate brachytherapy. J Urol 2013; 189: 1014-1018.

19. Bannowsky A, Bäurle L, Ataniyasov R et al. The chronological process of erectile function after low-dose rate prostate brachytherapy for localised prostate cancer. Aktuelle Urol 2011; 42: 252-255.

20. Chen RC, Basak R, Meyer AM et al. Association between choice of radical prostatectomy, external beam radiotherapy, brachytherapy, or active surveillance and patient-reported quality of life among men with localized prostate cancer. JAMA 2017; 317: 1141-1150.

21. Rhoden EL, Telöken C, Sogari PR et al. The use of the simplified International Index of Erectile Function (IIEF-5) as a diagnostic tool to study the prevalence of erectile dysfunction. Int J Impot Res 2002; 14: 245-250.

22. Ong WL, Hindson BR, Beaufort $C$ et al. Long-term erectile function following permanent seed brachytherapy treatment for localized prostate cancer. Radiother Oncol J Eur Soc Ther Radiol Oncol 2014; 112: 72-76.

23. Delaunay B, Delannes M, Salloum A et al. Orgasm after curietherapy with permanent iodine-125 radioimplants for localized prostate cancer. Prog Urol 2011; 21: 932-939.

24. Langley SEM, Soares R, Uribe J et al. Long-term oncological outcomes and toxicity in 597 men aged $\leq 60$ years at time of low-dose-rate brachytherapy for localised prostate cancer. BJU Int 2018; 121: 38-45.

25. Delage F, Perrouin-Verbe MA, Le Fur E et al. Erectile dysfunction evaluation after brachytherapy for low risk prostate adenocarcinoma: Prospective study of patients with a baseline IIEF5 > 16. Prog Urol 2015; 25: 68-74. 